\title{
Progress in the search for the optimum light source: squeezing experiments with a frequency doubler
}

\author{
H-A Bachor, T C Ralph, M S Taubman, A G White, C C Harb and \\ D E McClelland \\ Department of Physics, Faculty of Science, The Australian National University, ACT 0200 \\ Australia
}

\begin{abstract}
Real lasers show intrinsic noise well above the standard quantum noise limit. We review the properties of lasers and of techniques to suppress this noise. Experimental results of electro-optic feedback techniques and of squeezing in a second harmonic generator are presented. Finally we show that the optimum light source could well be a cascade of different cavities, each one performing a specific task in the noise suppression.
\end{abstract}

\section{Introduction}

The laser has been a revolutionary light source. It has provided access to the properties of light in an unprecedented way in regard to directionality, spectral purity, amplitude and phase accuracy.

The laser also revealed some of the limitations imposed by the quantum mechanical nature of the light, namely quantum noise in intensity and frequency which is a consequence of the uncertainty relationship. The consequences of quantum mechanics are noticeable in two technical limits: the intrinsic noise in intensity measurements, equivalent to shot noise [1] and the intrinsic noise in frequency measurements, expressed by the ShawlowTownes limit $[2,3]$. Real lasers, like those used in the experiments reported here, exhibit additional noise features. The combination of quantum and technical noise limits the sensitivity of optical measurements [4].

In this paper we describe the results from our attempts to surpass the standard quantum limits in search of the optimum light source. This paper deals exclusively with intensity noise, which is directly detectable and provides the technical limit in direct modulation techniques. We show the progress which has been made in understanding real lasers; the coupling of lasers with other optical systems, also known as cascaded systems; the use of frequency doublers for the generation of bright squeezed light, noise filtering in passive cavities and finally a proposal for intensity amplification without a loss of squeezing. It will be shown that the best realization of an optimum light source might well be a cascade of several components, each responsible for one particular task.

\section{Noise properties of a real laser}

A real laser combines the interaction of an active medium in a resonator and a source of energy for exciting the atoms, known as the pump. The technical noise is created through variations in these components such as vibrations changing the length of the resonator, intensity fluctuations of the pump, pressure or density fluctuations in the active medium. 
With sufficient experimental care these disturbances can be kept to a minimum and a laser emitting a single mode of light can be built. In our experiments we use Nd:YAG lasers, which presently provide the best combination of power and low noise performance $[5,6]$.

Any model of such a realistic laser involves the dynamics of a multi-level atom which in most cases is approximated by four levels, inside a cavity which is excited by the pump. We have to include the excitation and dephasing rates for the actual laser materials [7], in our case Nd:YAG.

The result of a theoretical model is the variance $V(\Omega)$ of the amplitude quadrature of the quantum state generated by the laser. It is normalized to the quantum noise limit which is defined as the noise level of a coherent state. How does this relate to observations in an experiment? There the laser beam is converted by a photodetector into a photocurrent and the fluctuations of the current are analysed. The variance determined in the model is equivalent to the experimentally determined normalized noise power measured with an electronic spectrum analyser at frequency $\Omega$;

$$
N(\Omega)=10 \log \left\{1-\frac{1}{\eta}\left[1-\frac{n(\Omega)-n_{\text {det }}(\Omega)}{n_{q l}-n_{\text {det }}(\Omega)}\right]\right\}=10 \log \mathrm{V}(\Omega)
$$

where $n(\Omega)$ is the optical noise power in the detected signal, $\eta$ is the efficiency of the detection system and $n_{\text {det }}(\Omega)$ is the electronic noise generated in the detector itself and is independent of incident intensity. $n_{q l}$ is the optical noise power corresponding to the standard quantum limit, and can be calculated by using the shot-noise formula

$$
n_{q l}=2 e I B
$$

where $I$ is the average power, $e$ the electric charge and $B$ the detection bandwidth. One possible experimental test for this calibration is a comparison with the noise generated by a thermal white light source with the same intensity and filtered to the same colour as the laser [8] or alternatively by comparing the output from two different detectors illuminated by equal fractions of the light, as shown in figure 1. In the latter case we make use of the fact that technical noise in the two outputs coming from one beamsplitter is correlated while the quantum noise is not correlated. Consequently, the sum of the two photocurrents shows both quantum and technical noise, and also squeezing, whilst the difference shows only the quantum noise [9].

In figure 2 we see the results for the case of a solid state $\mathrm{Nd:YAG} \mathrm{CW} \mathrm{laser} \mathrm{operating} \mathrm{well}$ above threshold $\left(P_{\text {pump }}=10 P_{t h r}\right)$. The most noticeable feature is the resonant relaxation oscillation (RRO), a consequence of the interaction between the atoms in the cavity and the field building up within it. This leads to a pronounced noise maximum at the frequency [10]

$$
\Omega_{R R O}=\sqrt{\frac{\frac{P_{\text {pump }}-1}{P_{\text {thr }}}-1}{\tau t_{c}}}
$$

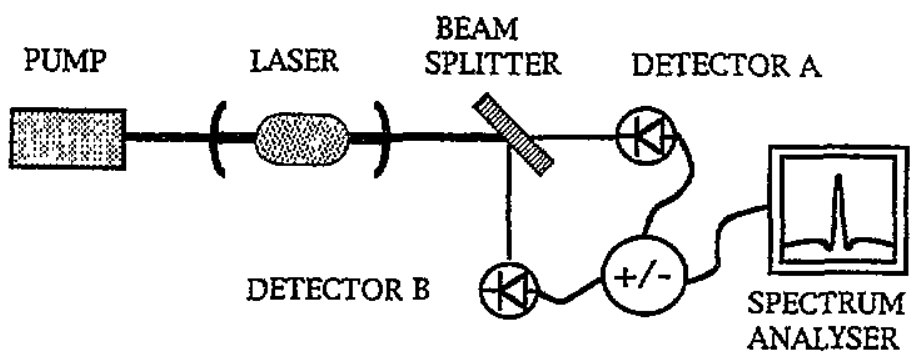

Figure 1. Schematic layout of a detection system for laser intensity noise. 


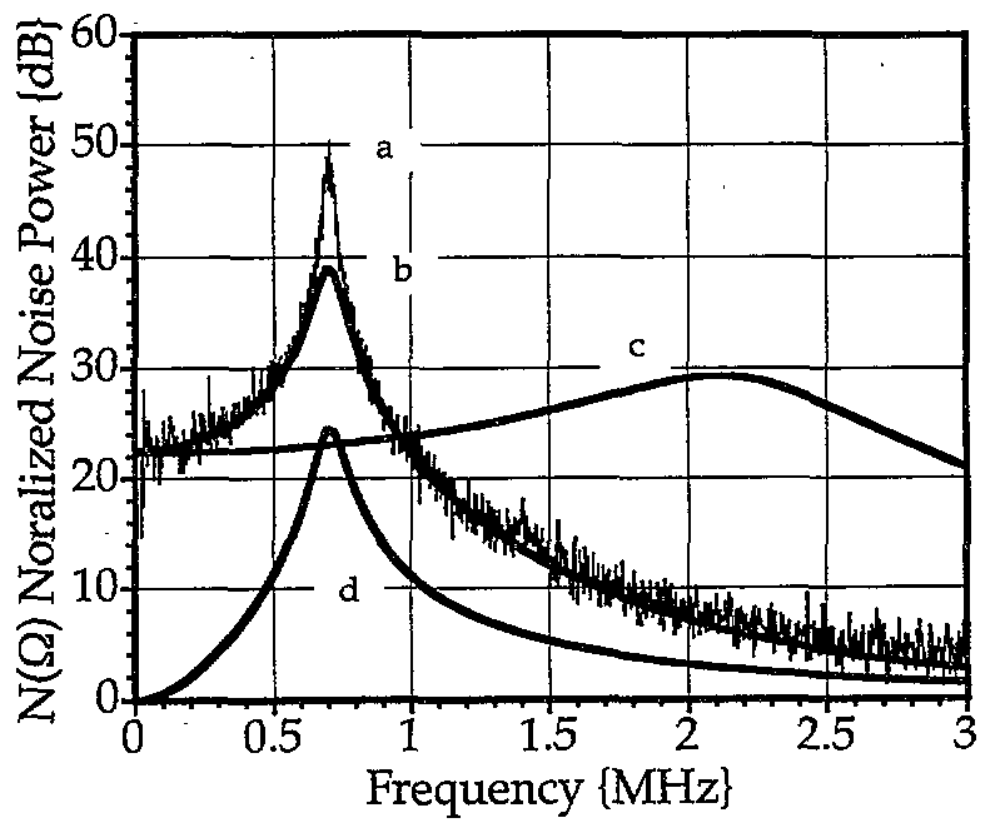

Figure 2. Laser noise spectra. (a) Experimental trace for a Nd:YAG laser operating about 10 times above threshold. (b) Theoretical prediction including $20 \mathrm{~dB}$ of broadband pump noise. (c) Shows theory if laser with noisy pump is operating 30 times above threshold. (d) Shows theory for laser 10 times above threshold with quantum noise limited pump.

where $\tau$ is the spontaneous lifetime of the upper lasing level, and $t_{c}$ is the cavity lifetime. Examples for practical lasers are: Nd:YAG $10^{5}-10^{6} \mathrm{~Hz}$, Erbium doped fibre lasers $10^{5}$ $10^{6} \mathrm{~Hz}$, Diode laser $10^{9} \mathrm{~Hz}$. At frequencies well above this oscillation $\left(\Omega \gg \Omega_{R R O}\right.$ ) the laser noise approaches the standard quantum limit. At these frequencies the output can be idealized as a coherent state [11]. The laser operates much like an ideal quantum mechanical oscillator. For frequencies at and below the oscillation $\left(\Omega<\Omega_{R R O}\right.$ ) the laser noise is above the standard quantum limit. It reflects the noise of the pump source. Trace (a) shows the noise spectrum of a diode laser pumped monolithic Nd:YAG laser, operating 10 times above the threshold. The diode laser pump contains about $20 \mathrm{~dB}$ of techical noise. Also shown (trace (b)) is the theoretical prediction, using a four-level model, for the parameters of this laser. Note that the performance of the laser at frequencies well above $\Omega_{R R O}$ is not influenced by the pump noise. An increase in the pump power (trace (c)) results in a shift of the relaxation frequency. The oscillation's peak is less pronounced and at a higher frequency. Trace (d) shows the noise spectrum predicted for the same condition as in trace (a) but with a quantum noise limited pump source.

Several techniques have been found to improve the noise performance. It is possible to suppress the noise by pumping the laser with a pump source operating below the standard quantum noise limit ( $Q N L)$, in particular when the pump is an electric current and not a beam of light [12]. For currents the QNL does not apply. Even some optically pumped lasers naturally run below the QNL when operated at extremely high pump powers [13] or when the rates of excitation and spontaneous emission are matched $[14,15]$. In the latter case having the excitation rate similar to one or more of the spontaneous rates in the pumping process leads to a 'self-regularization' of the pumping process. An example of the predicted 


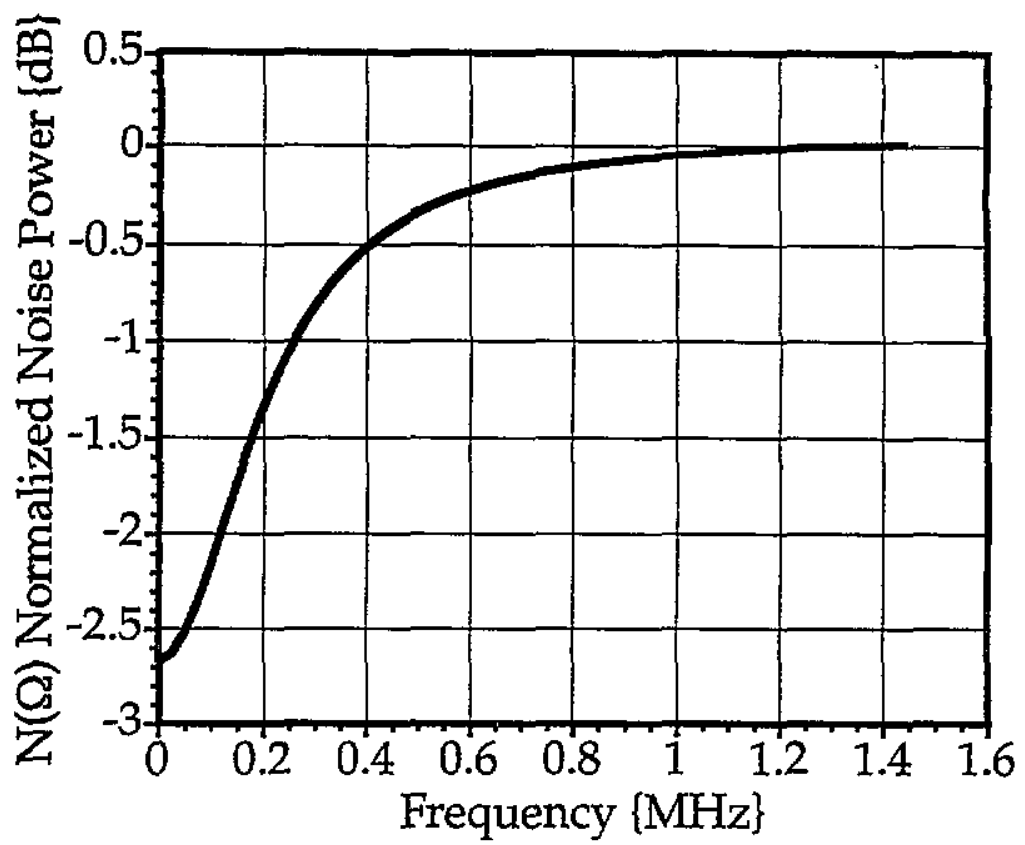

Figure 3. Theoretical laser spectrum for rate-matched laser. Parameters are chosen to be consistent with $\mathrm{Er}$ doped, Germanate glass, fibre laser operating 50 times above threshold.

noise spectrum for such a rate-matched laser is shown in figure 3 . In this case a fibre laser is modelled.

\section{Electro-optic feedback}

It is possible to reduce the relaxation oscillation noise using electro-optic feedback. For this purpose the fluctuations of the light are sampled using a beamsplitter. A fraction of the light is detected (figure 4) and the photocurrent from this detector is used to control either a modulator after the laser or the intensity of the pump. The remaining light transmitted by the beamsplitter is the useful output beam. Its properties can be determined with a second detector. Such a circuit, with a suitably designed feedback characteristic, will largely suppress classical fluctuations in the laser light. Figure 5 gives an example. The gain and phase of the feedback loop are shown in part (i) and (ii) of the figure. The measured noise level with and without the feedback is shown is part (iii). The details of actually building this feedback system and how to avoid time delays and phase lags are intricate and a skill by itself $[16,17]$.

Electro-optic feedback with direct detection cannot suppress quantum noise. This is a consequence of the correlation properties of the two output beams of the beamsplitter. The quantum noise component of the noise is not correlated and thus cannot be controlled. The current from the detector inside the feedback loop will actually show fluctuations well below the standard quantum limit. This has caused considerable interest in the nature of the light illuminating this detector [18-21]. This light is not a freely propagating wave of light. It is in a state which is conditional on the feedback loop. In a quantum mechanical description it does not satisfy the conventional commutation rule [18]. The sensible question to ask is whether this beam can be used to perform any measurement with a signal-to-noise ratio 
MODULATOR

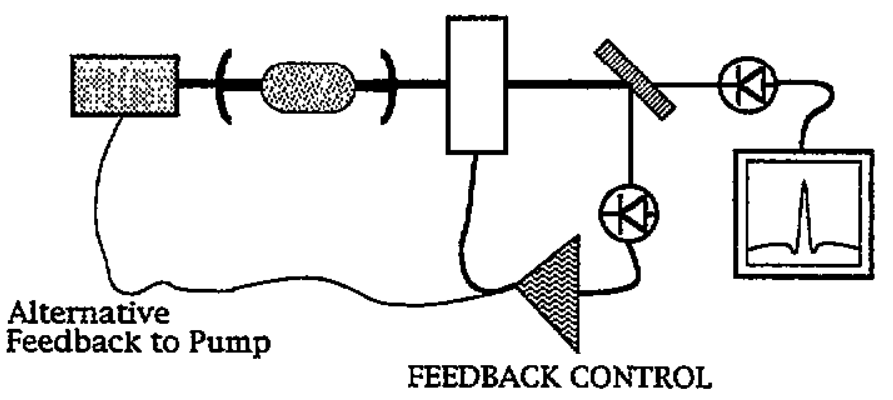

Figure 4. Schematic layout of electro-optic intensity control by feedback with direct detection.
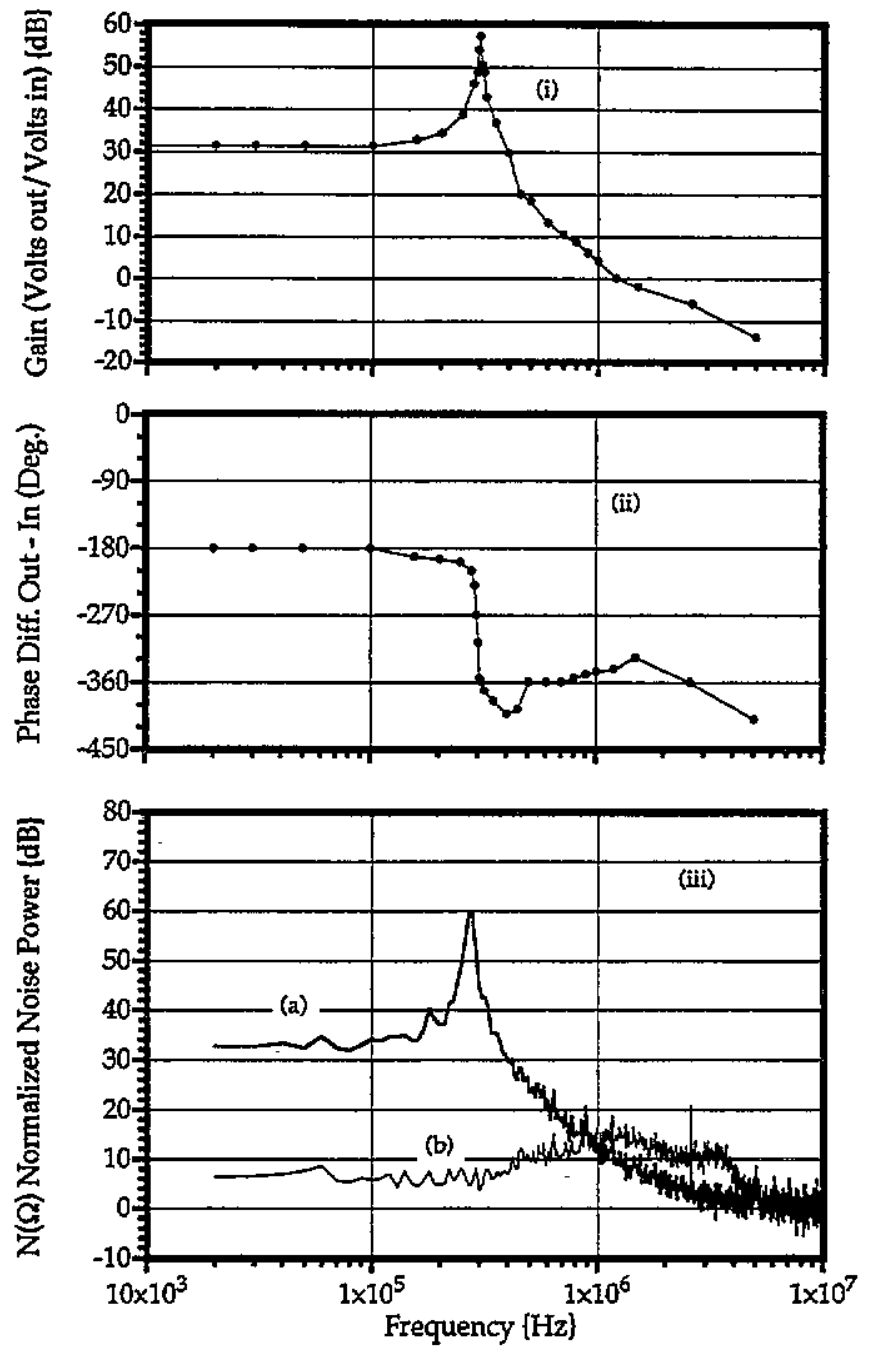

Figure 5. Experimental noise spectra from a Nd:YAG laser with electro-optic intensity control. Part (i) shows the gain of the feedback loop. Part (ii) shows the phase shift of the feedback loop. Part (iii) (a) shows the noise spectrum without feedback. (iii) (b) noise spectrum with feedback. 
better than can be achieved with a quantum noise limited beam. The answer is a clear no since both the signal and the noise are equally affected by the feedback [22]. All possible schemes using a beamsplitter will achieve, at best, the performance of a QNL system [24].

There is actually a penalty to be paid for the noise suppression: the feedback will increase the minimum achievable noise level, in particular when the feedback gain is high. The suppression of noise is limited to this increased level. This can be seen in part (iii) of figure 5 for frequencies below $0.3 \mathrm{MHz}$. Here the noise level after feedback is still about $6 \mathrm{~dB}$ above the standard quantum limit despite the perfectly well behaved control system. This penalty is a consequence of having detected only a quarter of the light for the feedback control and thereby imposing the uncorrelated quantum noise from the feedback detector onto the output beam [22].

Improvements on feedback with direct detection can only be made by replacing the beamsplitter by an optical nonlinear component, such as a Kerr medium or a frequency doubler [23]. In these cases the direct detection is replaced by a quantum nondemolition measurement which, coupled with feedback, can lead to laser light with sub-QNL noise $[25,26,23]$.

\section{Squeezing of intensity noise}

An alternative is to use the nonlinear medium to generate bright, amplitude squeezed light directly. Second harmonic generation (SHG) was one of the first processes which was explored for squeezing [27] and gradual improvement of the materials and techniques [28-30] have recently resulted in reliable noise suppression [31,32]. There are a number of possible schemes using either SHG in a second cavity, outside the laser (passive SHG), or in the laser itself (active SHG) [33]. The system can be either singly resonant, for the fundamental wavelength, or it can involve a system which is simultaneously resonant for both the fundamental and the SHG light. In the latter case the squeezing effect is enhanced at certain frequencies determined by the instabilities and the dynamics of the doubly resonant system [34]. Either the fundamental or the SHG light can be squeezed, usually one particular system will be optimized for only one of the two beams. Table 1 summarises these various possibilities [35]. The table lists the predicted noise suppression $\left(V_{\min }\right.$ between 0 and 1$)$ and the detection frequency $\left(\Omega_{\min }\right.$ in multiples of the effective cavity linewidth of the squeezing cavity) at which this squeezing would occur. The table gives the theoretical limits for ideal systems and the results predicted for real systems based on current technology, which include the losses, linewidths and dephasing rates for a practical laser system such as Nd:YAG. Real systems show significantly less noise suppression. In particular, the predicted squeezing at very low frequencies $\left(\Omega_{\min }=0\right)$ is not observable when real lasers are used. Best squeezing will be close to the effective cavity linewidth of the external SHG cavity. It should be noted that the active systems are the simplest to build but the most unlikely systems to produce squeezing. The squeezing will not be observable in conventional active systems, because it will be eliminated by the dephasing rates. Only the doubly resonant active system for suppression of the second harmonic light shows some promise. However, squeezing would require extremely high pump powers, orders of magnitude larger than those obtainable today.

Out of these options, passive frequency doublers with singly resonant cavities are so far the most reliable systems for noise suppression [31,32]. We report on experiments with this arrangement which is shown in figure 6. The sHG material is $\mathrm{MgO}$ doped $\mathrm{LiNbO}_{3}$ which is shaped into a monolith, this means the crystal is also the resonator. The end surfaces are curved, polished [36] and coated to be dielectric high reflective mirrors [37]. A diode 
Table 1. Maximum noise suppression $V_{m i n}$ and frequency $\Omega_{m i n}$ at which it occurs. $\Omega_{\min }$ is in units of effective cavity linewidth $\gamma_{\text {eff }}$. The table distinguishes between the cases for singly and doubly resonant systems squeezing either the fundamental or the frequency doubled light. The theoretical limit considers a single ended, lossless device. The laser limit considers pump noise and dephasing rate of a Nd:YAG laser as well as cavity linewidth, nonlinear coupling, losses and reflectivities of a monolithic SHG available today.

\begin{tabular}{|c|c|c|c|c|c|c|c|c|}
\hline \multirow[b]{3}{*}{ Mode } & \multicolumn{4}{|c|}{ Passive } & \multicolumn{4}{|c|}{ Active } \\
\hline & \multicolumn{2}{|c|}{ Theoretical limit } & \multicolumn{2}{|c|}{ Laser limit } & \multicolumn{2}{|c|}{ Theoretical limit } & \multicolumn{2}{|c|}{ Laser limit } \\
\hline & $2 \omega$ & $\omega$ & $2 \omega$ & $\omega$ & $2 \omega$ & $\omega$ & $2 \omega$ & $\omega$ \\
\hline $\begin{array}{l}\text { Singly } \\
\text { resonant } \\
\text { at } \omega\end{array}$ & $\begin{array}{l}V_{\min }=\frac{1}{9} \\
\Omega_{\min }=0\end{array}$ & $\begin{array}{l}V_{\min }=\frac{2}{3} \\
\Omega_{\min }=0\end{array}$ & $\begin{array}{l}V_{\min } \simeq 0.5 \\
\Omega_{\min } \simeq 1.0 \gamma_{e f f}\end{array}$ & $\begin{array}{l}V_{\min } \simeq 0.9 \\
\Omega_{\min } \simeq 1.2 \gamma_{e f f}\end{array}$ & $\begin{array}{l}V_{\min }=\frac{1}{2} \\
\Omega_{\min }=0\end{array}$ & $\begin{array}{l}V_{\min }=\frac{1}{2} \\
\Omega_{\min }=0\end{array}$ & $\begin{array}{l}\text { No } \\
\text { squeezing } \\
\text { Dephasing }\end{array}$ & $\begin{array}{l}\text { No } \\
\text { squeezing } \\
\text { Dephasing }\end{array}$ \\
\hline $\begin{array}{l}\text { Doubly } \\
\text { resonant }\end{array}$ & $\begin{array}{l}V_{\min }=0 \\
\Omega_{\min } \neq 0\end{array}$ & $\begin{array}{l}V_{\min }=0 \\
\Omega_{\min } \neq 0\end{array}$ & $\begin{array}{l}V_{\min } \simeq 0.1 \\
\Omega_{\min } \simeq 1.5 \gamma_{e f f}\end{array}$ & $\begin{array}{l}V_{\min } \simeq 0.3 \\
\Omega_{\min } \simeq 0.8 \gamma_{e f f}\end{array}$ & $\begin{array}{l}V_{\min }=0 \\
\Omega_{\min }=0\end{array}$ & $\begin{array}{l}V_{\min }=0 \\
\Omega_{\min } \neq 0\end{array}$ & $\begin{array}{l}\text { No } \\
\text { squeezing } \\
\text { High pump }\end{array}$ & $\begin{array}{l}\text { No } \\
\text { squeezing } \\
\text { + dephasing }\end{array}$ \\
\hline
\end{tabular}

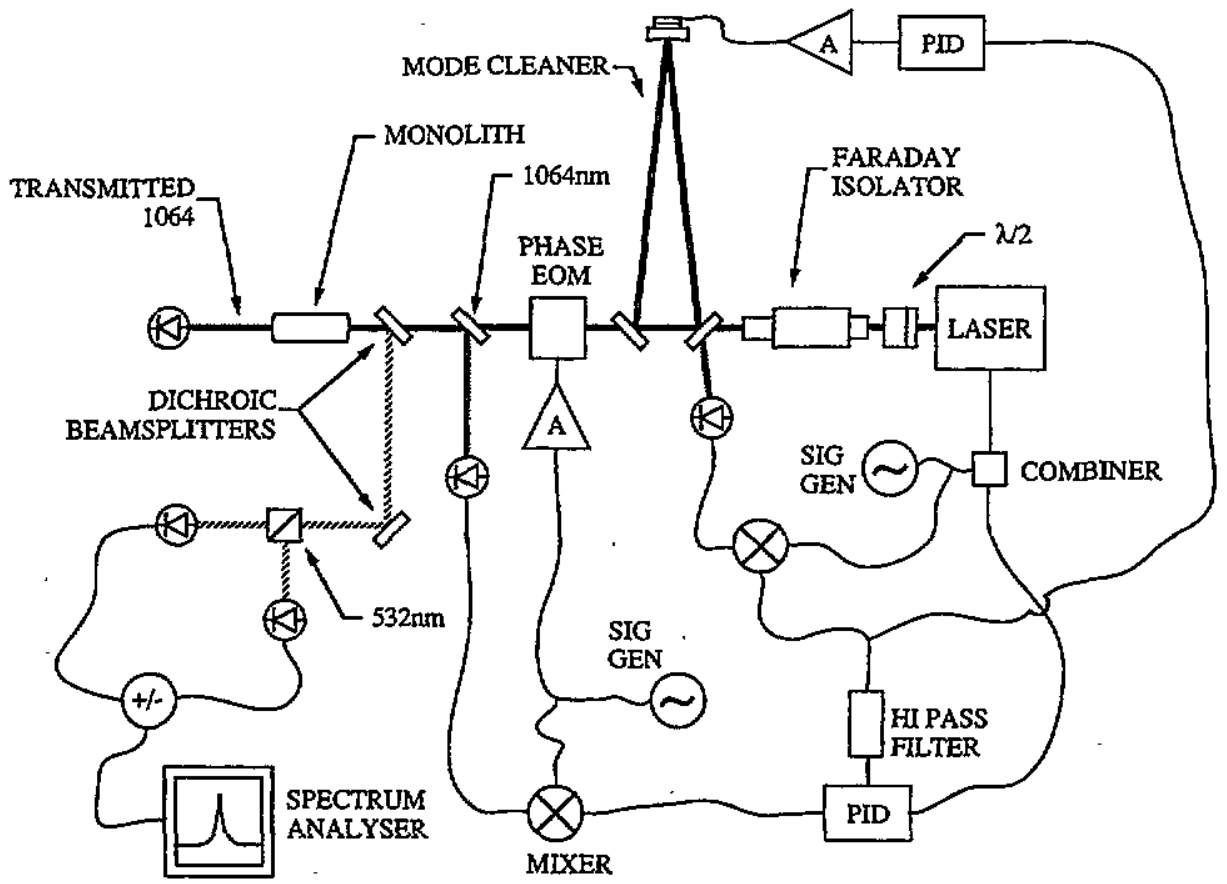

Figure 6. Layout of the experiment for the generation of squeezed light by second harmonic generation. A mode-cleaner cavity filters the noise from the $\mathrm{CW}$ Nd:YAG laser. The second harmonic light is detected by a balanced detector. The electronic system for locking the laser frequency to the resonance of the SHG monolithic cavity and the mode cleaner is shown in detail.

pumped CW Nd:YAG laser operating at $1064 \mathrm{~nm}$ is locked to this resonator. The SHG has a conversion efficiency from $1064 \mathrm{~nm}$ to $532 \mathrm{~nm}$ of over $50 \%$. The squeezed sHG light $(532 \mathrm{~nm})$ is separated with a dichroic mirror and is detected by a balanced pair of detectors. The difference between the two photocurrents (minus trace) defines the standard quantum 


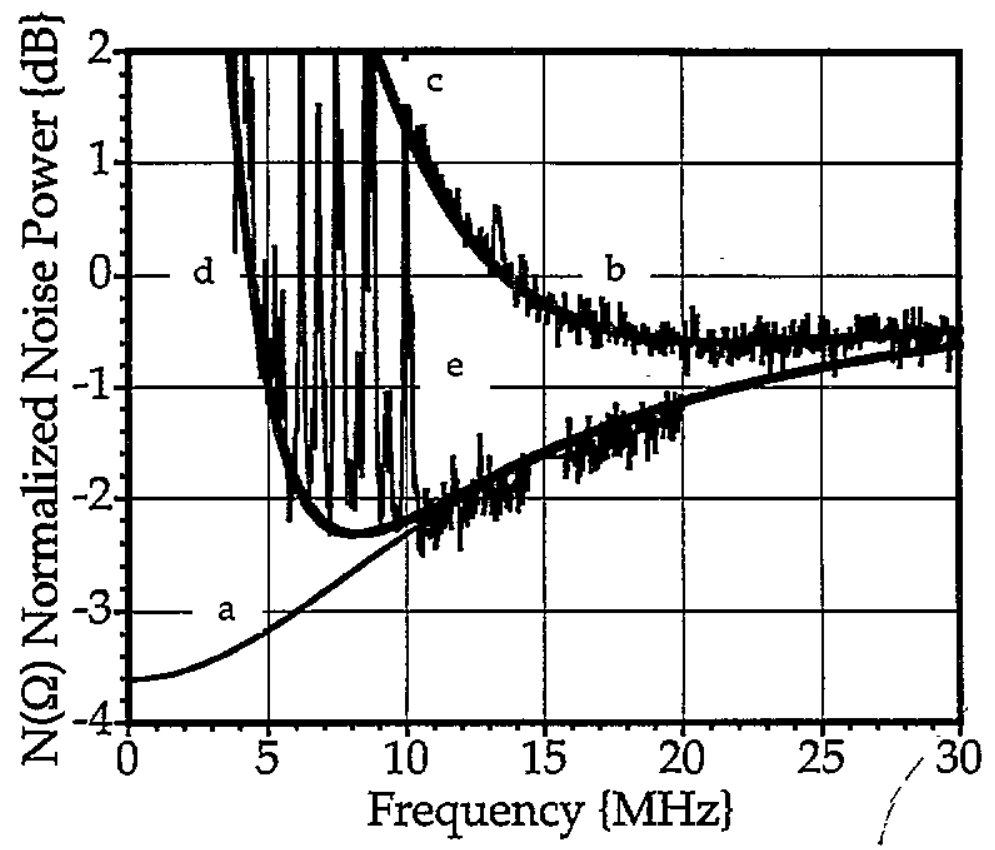

Figure 7. Noise spectra for second harmonic generation. (a) Theoretical prediction for SHG with a quantum noise limited input beam. Parameters matched to experimental sHG crystal. (b) Experimental data of SHG driven by laser, corrected for detector quantum efficiency $(\eta=0.65$ ) and electronic noise. (c) Theoretical prediction including the noisy laser pump. (d) Theoretical prediction if a mode cleaning cavity is placed between the laser and the sHG crystal. The parameters are identical to those in $(c)$. (e) Experimental data for system with a mode cleaner.

limit; the sum of the currents (plus trace) shows both squeezing and classical noise. The technical difficulties are in maintaining the optimum phase matching temperature; mode matching of the laser into the SHG resonator and keeping the laser-resonator detuning fixed at the optimum level [32].

Figure 7 shows the results for a scan of the detection frequency. Trace (a) is the initial model calculations of the squeezing for a doubler illuminated by a coherent state [30]. The realistic values for the coupling and the cavity parameters are used. Trace (b) shows the experimental results. The agreement at large detection frequencies is reasonable, but the prediction of good noise suppression at low frequencies is clearly wrong. Here the noise properties of the real laser dominate. A second model, based on the formalism of cascaded systems $[38,39,32]$, was developed which simultaneously described the laser and the SHG resonator. It is shown in trace (c) and is in excellent agreement with the measured results. The model can be used to include a third, passive, empty cavity with moderate finesse and narrow linewidth which is placed between the laser and the SHG (see figure 6). This cavity acts as a spatial mode cleaner and, more importantly, as a low-pass filter for the laser noise. The low-frequency noise outside the mode-cleaner cavity linewidth is reflected. The mode cleaner has to be kept in lock with the laser frequency by feedback control. The prediction of this three-cavity model is shown in trace (d) of figure 7. The squeezing extends to lower frequencies and is larger. The corresponding experimental results are shown in trace (e). The agreement is excellent for frequencies above $11 \mathrm{MHz}$. Below this we clearly see 


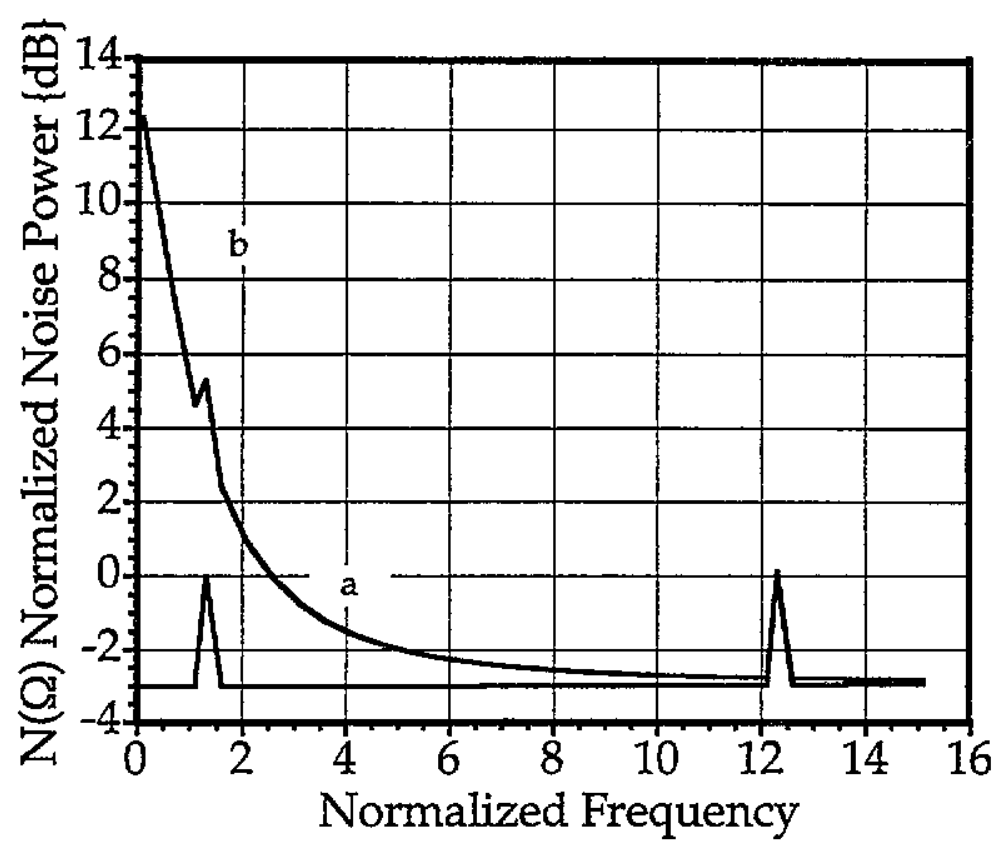

Figure 8. Calculated noise spectra for a narrow linewidth laser amplifier with broadband squeezing input. Frequency normalized by the laser amplifier bandwidth. The input contains amplitude modulation signals within and well above the bandwidth. (a) Input signal. (b) Output signal.

yet another source of technical noise, most likely due to acoustic resonances in the SHG crystal.

\section{Future options}

As can be seen from this example, squeezing is already a useful tool. Given further technical improvements, we can expect noise suppression of more than $6 \mathrm{~dB}$ (fourfold) as shown in table 1 for a doubly resonant system. Similarly large suppression has already been seen with OPOs for vacuum squeezed light $[40,41]$.

However, squeezed light is known to be rather fragile [8]. What is needed are techniques for modifying the intensity of the laser beam without losing the noise supprossion. Conventional attenuation or amplification is useless, since it reduces the squeezing [42, 43]. For both these situations we have found theoretical solutions, which we wish to test in future experiments. For the attenuation we can use the electro-optic feedback with direct detection described above, but this time with a positive, rather than negative (stabilizing) feedback. The squeezed light can be interpreted as noise with negative correlations and the two output beams from the beamsplitter will maintain these correlations. Thus the feedback loop is able to either reduce or enhance this component of the noise in the ouput beam. For certain levels of positive feedback while the noise in the feedback loop is increased, that in the output beam is reduced. In this manner it is possible to transfer most of the squeezing to the output beam, which has a reduced intensity [22]. Note that this device cannot generate a noise suppression larger than that of the input.

Similar attenuation can be achieved by reffecting the squeezed light off an empty 


\section{Acknowledgments}

This work was supported by grants from the Australian Research Council. We wish to thank B Brown at ANU, the Laser Zentrum Hannover and CSIRO Optical Technology, Lindfield for their technical support.

\section{References}

[1] Saleh B E A 1978 Photoelectron Statistics (Berlin: Springer)

[2] Schawlow A L and Townes C H 1958 Phys. Rev. 11940

[3] Hempstead R D and Lax M 1967 Phys. Rev. 161350

[4] Yamamoto Y, Machida S, Saito S, Imoto N, Yaganawa T, Kitigawa M and Bjoerk G 1990 Quantum mechanical limit in optical precision measurement and communication Progress in Optics vol XXVIII ed E Wolf

[5] Kane T J, Nilsson A C and Byer R L 1987 Opt. Lett. 12175

[6] Köpke I PhD Thesis Hannover

[7] Koechner W 1990 Solid State Lasers (Berlin: Springer)

[8] Kimble H J 1992 Phys. Rep. 219227

[9] Walls D F and Milburn G J 1993 Quantum Optics (Berlin: Springer)

[10] Yariv A 1989 Quantum Electronics 3rd edn (New York: Wiley)

[11] Glauber R J 1962 Phys. Rev. 1312766

[12] Yamamoto Y N and Machida S 1986 Phys. Rev. A 355114

[13] Kobolov M I, Giacobino E, Fabre C and Davidovich L 1993 Phys. Rev. A 471431

[14] Ritsch H, Zoller P, Gardiner C W and Walls D F 1991 Phys. Rev. A 443361

[15] Ralph T C and Savage C 1991 Phys. Rev. A 447809

[16] Hough J, Ward H, Kerr G A, Mackenzie N L, Meers B J, Newton G P, Robertson D I, Robertson N A and Schilling R 1991 The Detection of Gravitational Waves ed D G Blair (Cambridge: Cambridge University Press)

[17] Harb C, Gray M B, Bachor H-A, Schilling R, Rottengatter P, Freitag I and Welling H 1994 IEEE J. Quantum Electronics

[18] Shapiro J H, Saplakoglu G, Ho S-T, Kumar P, Saleh B E A and Teich M C 1987 J. Opt. Soc. Am. B 4 1604

[19] Mertz J, Heidmann A and Fabre C 1991 Phys. Rev. A 443229

[20] Masalov A V, Putilin A A and Vasilyev M V 1994 J. Mod. Opt. 411941

[21] Youn S-H, Jhe W, Lee J-H and Chang J-S 1994 J. Opt. Soc. Am. B 11102

[22] Taubman M S, Wiseman H, McClelland D E and Bachor H-A 1995 Quantum effects of intensity feedback J. Opt. Soc. Am. B submitted

[23] Wiseman H M, Taubman M S and Bachor H-A 1995 Feedback-enhanced squeezing in second harmonic generation Phys. Rev. A at press

[24] Wiseman H M and Milbum G J 1993 Phys. Rev. A 471652

[25] Grangier P, Poizat J-Ph, Grelu P, Castelli F, Ligiato L A and Sinatra A 1994 Quantum Optics VI ed D F Walls and J D Harvey (Berlin: Springer) pp 123-33

[26] Yamamoto Y, Imoto N and Machida S 1986 Phys. Rev. A 333243

[27] Pereira S F, Xiao Min, Kimble H J and Hall J L 1988 Phys. Rev. A. 384931

[28] Sizmann A, Horowicz R J, Wagner G and Leuchs G 1990 Opt. Commun. 80138

[29] Kürz P, Paschotta R, Fiedler K, Sizmann A and Mlynek J 1993 Europhys. Lett. 24449

[30] Collett M J and Levien R B 1991 Phys. Rev. A 425068

[31] Paschotta R, Collett M, Kürz P, Fiedler K, Bachor H-A and Mlynek J 1994 Phys. Rev. Lett. 72 3807-10

[32] Ralph T C, Taubman M S, White A G, McClelland D E and Bachor H-A 1995 Squeezed light from second harmonic generation; experiment versus theory $O p t$. Lett. at press

[33] Sizmann A, Schack R and Schenzle A 1990 Europhys. Lett. 13 109-15

[34] Collett M J and Walls D F 1985 Phys. Rev. A 322887

[35] White A G, Ralph T C and Bachor H-A work in progress, Private communication

[36] The supersmooth surfaces of the SHG monolith were polished by CSIRO Optics Technology, Lindfield, Australia.

[37] The low loss coatings on the SHG monolith were provided by the Laser Zentrum Hannover, Germany

[38] Carmichael H J 1993 Phys. Rev. Lett. 702273

[39] Gardiner C W 1993 Phys. Rev. Lett. 702269 
[40] Polzik E S, Cari J C and Kimble H J 1992 Phys. Rev. Lett. 683020

[41] Similar results were recently achieved by Mueller T Pereira S F and Breitenbach G Private communication

[42] Haus H A and Mullen J A 1962 Phys. Rev. 1282407

[43] Caves C M 1982 Phys. Rev. D 261817

[44] Levenson M D, Shelby R M and Perlmutter S H 1985 Opt. Letr. 10514

[45] Ralph T C and Bachor H-A 1995 Noiseless amplification of squeezed light using a standard laser amplifier Opt. Commun. submitted 